\title{
In vivo assessment of myelination by phase imaging at high magnetic field
}

\author{
Gregory A. Lodygensky ${ }^{\text {a,c }}$, José P. Marques ${ }^{\text {b,d,* }}$, Rajika Maddage ${ }^{\text {b }}$, Elodie Perroud ${ }^{c}$, Stéphane V. Sizonenko ${ }^{c}$, \\ Petra S. Hüppi ${ }^{c}$, Rolf Gruetter ${ }^{\text {b, d,e }}$ \\ a Pediatrics, Pediatric and Neonatal ICU, University of Geneva, Geneva 14, Switzerland \\ ${ }^{\mathrm{b}}$ Laboratory for Functional and Metabolic Imaging, Ecole Polytechnique Fédérale de Lausanne, Lausanne, Switzerland \\ c Pediatrics, Division of Child Growth \& Development, University of Geneva, Geneva, Switzerland \\ d Department of Radiology, University of Lausanne, Lausanne, Switzerland \\ e Department of Radiology, University of Geneva, Geneva, Switzerland
}

\section{A R T I C L E I N F O}

\section{Article history:}

Received 30 May 2011

Revised 21 September 2011

Accepted 21 September 2011

Available online 1 October 2011

\section{Keywords:}

Phase contrast

MRI

Myelin quantification

Phase imaging

Dysmyelination

Shiverer

\begin{abstract}
A B S T R A C T
The present study evaluated the potential of using the phase of $\mathrm{T}_{2}{ }^{*}$ weighted MR images to characterize myelination during brain development and pathology in rodents at $9.4 \mathrm{~T}$. Phase contrast correlated with myelin content assessed by histology and suggests that most contrast between white and cortical gray matter is modulated by myelin. Ex vivo experiments showed that gray-white matter phase contrast remains unchanged after iron extraction. In dysmyelinated shiverer mice, phase imaging correlated strongly with myelin staining, showing reduced contrast between white and gray matter when compared to healthy controls. We conclude that highresolution phase images, acquired at high field, allow assessment of myelination and dysmyelination.
\end{abstract}

(c) 2011 Elsevier Inc. All rights reserved.

\section{Introduction}

The assessment of myelination in healthy subjects and following injury in humans and in animal models is one of the ways to determine the degree of cerebral integrity or the degree of injury in case of white matter disease like multiple sclerosis. In newborns, myelination of the posterior limb of the internal capsule, when assessed at term equivalent, has been shown to be a very robust predictor of motor impairment (Cowan and de Vries, 2005). A simple technique that would access the degree of myelination of white matter would be very useful in mature animal models of white matter injury such as multiple sclerosis and in immature animal models of white matter injury such as periventricular leukomalacia as well as in human studies. There are various methods available: one approach consists of quantification of myelin bond and free water fractions by a multiexponential analysis of the $T_{2}$ decay (Beaulieu et al., 1998; MacKay et al., 1994; Whittall and MacKay, 1989; Lancaster et al., 2003). This is generally performed using a CarrPurcell-Meiboom-Gill (CPMG) or a Turbo Spin-Echo sequences with multiple echo times (Beaulieu et al., 1998). Due to specific absorption rate (SAR) (especially at high fields) and timing constraints, the $\mathrm{T}_{2}$

\footnotetext{
* Corresponding author at: EPFL SB IPSB LIFMET, CH F1 612 (Bâtiment CH), Station 6, 1015 Lausanne, Switzerland.

E-mail address: jose.marques@epfl.ch (J.P. Marques).
}

decay curve is often not fully sampled for an accurate quantification. Recently, it has been shown that myelin water fraction can also be measured using a multi-exponential analysis of the $\mathrm{T}_{2}{ }^{*}$ decay in multislice multiple echo gradient echo sequence (Du et al., 2007; Hwang et al., 2010). The resulting myelin water fractions are highly dependent on the constraints introduced on the multi-exponential fitting procedure (Hwang et al., 2010) and suffer from significant inaccuracies in regions close to air-tissue interfaces. An alternative approach of a multicomponent relaxation analysis using multicomponent driven equilibrium single pulse observation of T1 and T2 (mcDESPOT) (Deoni et al., 2008) was applied successfully on infants from 3 to 11 months to quantify myelination (Deoni et al., 2011). Magnetization transfer represents an alternative approach to quantify myelin with new approaches refining its modelization by characterizing individually the relaxation and exchange rates of the free water and macromolecular protons (Ou et al., 2009). It has also been shown that the diffusion components perpendicular to the main eigenvector of water diffusion in DTI acquisitions, i.e. radial diffusivity (average of lambda 2 and 3 in diffusivity maps) in animal models is a surrogate marker of myelin integrity (Song et al., 2002) with the decrease of the diffusivity in this direction being related to the degree of myelination. Although the radial diffusivity can be affected by myelination, it has been shown that in developing rodents the radial diffusivity shows changes before the establishment of myelination (Lodygensky et al., 2010), reflecting the underlying microstructural maturation rather than the myelination process. Furthermore, high resolution DTI is time consuming and challenging to obtain 
due to the high gradient demands. In conclusion, although several promising approaches exist to characterize the degree of myelination, they suffer from practical limitations.

On the other hand, MR phase imaging, acquired with a standard gradient recalled echo, has been shown to emphasize contrast between gray and white matter (Duyn et al., 2007) when compared to conventional magnitude imaging. Phase contrast was shown to be affected by tissue lipid and iron content (Duyn et al., 2007; Fukunaga et al., 2010) and the local microstructure (He and Yablonskiy, 2009). The effect of cerebral blood volume has been demonstrated to have a minor, if any, impact on the phase contrast observed between white and gray matter (Lee et al., 2010a; Marques et al., 2009). While phase imaging offers an improved contrast between tissues, it should be taken into account that this information is non-local. It represents, at least to a large extent, the convolution of a magnetic susceptibility distribution with the magnetic field generated by magnetic dipole (Marques and Bowtell, 2005; Salomir et al., 2003). Such property makes the contrast observed between two tissues dependent on their orientation relatively to the main magnetic field and to extend beyond their boundaries (Schafer et al., 2009). It is therefore not surprising that many groups have centered their attention on how to calculate the underlying magnetic susceptibility (Cheng et al., 2009; de Rochefort et al., 2008, 2010; Liu et al., 2009; Shmueli et al., 2009), based on the measured phase images. Recently two independent groups have observed that the susceptibility associated with white matter was anisotropic (Lee et al., 2010b; Liu, 2010), with Liu's work introducing the concept of susceptibility tensor imaging. Recently the same group (Liu et al., 2011) has shown in ex vivo mice brain that most of the contrast observed both in phase images and in the calculated susceptibility maps was significantly reduced in shiverer mice in respect to the control group. Shiverer mice are a model of congenital dysmyelination characterized by a $98 \%$ reduction of myelin basic protein with a subsequent alteration of myelin compaction and reduction of myelin layers (Chernoff, 1981; Privat et al., 1979). This animal model was used to show the importance of the contribution of myelin towards the observed contrast.

The aim of this study was to determine in vivo to what extent the phase difference observed between gray and white matter reflects (or is modulated by) the degree of myelination by studying rat pups during development and comparing normal and shiverer mice populations.

\section{Material and methods}

\section{Animal preparation}

Animal handling procedures were approved by the Office Vétérinaire Cantonal (Geneva) in accordance with Swiss Federal Laws. Postnatal day 3 (P3) $(n=3), 4$ (P4) $(n=2), 15$ (P15) $(n=3)$, and 40 $(\mathrm{P} 40)(\mathrm{n}=3)$ Wistar rats were anesthetized with isoflurane $(0.5-2 \%)$ and placed in a neonatal or an adult custom-built MRI holder in ertalyte. Two birdcages of 2.5 and $3.5 \mathrm{~cm}$ in diameter were used (Stark Contrast, Erlangen, Germany) depending on the age of rat pups.

Five weeks old male mice $(n=5)$ homozygous for the shiverer spontaneous mutation $\mathrm{MBP}^{\text {shi }}$ and 5 male age matched controls $\mathrm{C} 3 \mathrm{HeB} / \mathrm{FeJ}$ mice (The Jackson Laboratory, Bar Harbor, Maine, USA) were also included in this study. Imaging was performed in a custombuild ertalyte MRI compatible holder with a quadrature coil actively decoupled with an external Alderman transmit coil.

Food and water were provided to the dam under evaluation ad libitum. Mice were anesthetized with isoflurane (0.5-2\%) during the imaging protocol. Body temperature was monitored and kept stable. Respiration rate was monitored using a pressure pillow placed under the abdomen.

\section{Magnetic resonance imaging}

All MRI studies were acquired on an actively shielded $9.4 \mathrm{~T} / 31 \mathrm{~cm}$ bore magnet (Magnex Scientific, Abingdon, UK) with a $12 \mathrm{~cm}$ gradient set $(400 \mathrm{mT} / \mathrm{m}$ in $120 \mu \mathrm{s})$. The magnet, gradient coil and gradient power supply were run with an Inova console (Varian Associates, Palo Alto, CA).

Magnetic field homogeneity was adjusted using FASTMAP (Gruetter, 1993) to minimize dephasing and image distortions. A standard coronal gradient-echo sequence was used with $\mathrm{TR} / \mathrm{TE}=900 / 14 \mathrm{~ms}$, matrix size $256 \times 256$ with a square FOV of 16 to $27 \mathrm{~mm}$ depending on the age, a slice thickness of $0.5 \mathrm{~mm}$ (P3 20-32, P4 32, P15 32, P21 32, P40 38 slices), a spectral width of $50 \mathrm{\prime} 000 \mathrm{~Hz}$ (resulting in a sampling window of $5.12 \mathrm{~ms}$ ), 8 averages and a total scan time $30 \mathrm{~min}$ and $50 \mathrm{~s}$. Chosen parameters were similar to an in vivo rat brain study performed at $14.1 \mathrm{~T}$ (Marques et al., 2009), with the echo time and acquisition times being smaller to minimize respiration induced artifacts. Coronal diffusions images were acquired on mice only with a spin-echo sequence using a $\mathrm{TR} / \mathrm{TE}=2000 / 36 \mathrm{~ms} 2$ averages, a slice thickness $=0.5 \mathrm{~mm}$, an inplane resolution of $0.250 \times 0.125 \mathrm{~mm}$ zero filled to $0.125 \times 0.125 \mathrm{~mm}$. The delay between application of the gradient pulses was set to $21 \mathrm{~ms}$ and had a duration of $7 \mathrm{~ms}, b$ value amplitudes $=0$ and $838 \mathrm{~s} / \mathrm{mm}^{2}$. Two sets of 6 directions, chosen in an origin-symmetric fashion (Jara and Wehrli, 1994), were acquired. The total acquisition time of this protocol was $59 \mathrm{~min}$ and $44 \mathrm{~s}$.

\section{Ex vivo studies}

Four ex vivo P30 rat brains, 2 control and 2 iron-extracted by reductive dissolution (Fukunaga et al., 2010) were used for this experiment. A custom-build inductively coupled solenoid coil perpendicular to B0, which served both as a RF transmitter and receiver and a conventional birdcage coil was used. The same gradient-echo sequence was used with the same TR/TE, matrix and slice thickness as aforementioned, and a FOV of $16 \mathrm{~mm}$. Due to geometrical constraints, when scanning with the solenoid coil, the anterior posterior direction of the rat brain was positioned perpendicular to the main magnetic field as opposed to the parallel orientation of the birdcage coil.

\section{Histology}

After MR imaging, the rat received a lethal injection of ketamine and lidocaine and was transcardially perfused with saline followed by $4 \%$ paraformaldehyde. Brains were extracted and immersion fixed in $4 \%$ paraformaldehyde for $24 \mathrm{~h}$ at $4{ }^{\circ} \mathrm{C}$ and then cryoprotected by immersion in 30\% sucrose. $50 \mu \mathrm{m}$ thick coronal sections were cut with a cryostat and conserved in a cryoprotectant solution (30\% ethylene glycol, $0.03 \mathrm{M} \mathrm{PO4,15 \%}$ sucrose) until staining.

\section{Myelin staining}

Free floating brain sections were stained with Black Gold II myelin staining kit according to the manufacturer instructions (Millipore, Billerica, MA) (Schmued et al., 2008) and scanned at 20× with the Mirax Midi (Zeiss, Göttingen, Germany). A mean optical densitometry was calculated on matching regions of interest with the software Mercator (Explora Nova, La Rochelle, France).

\section{Iron extraction}

Iron extraction of a P30 Wistar rat brain was achieved by reductive dissolution using a PBS solution with $2 \mathrm{mM}$ sodium dithionite (Kremer Pigmente GmbH \& Co. KG) and $1 \mathrm{mM}$ of deferoxamine (Sigma-Aldrich, St Louis, MO), an iron chelating agent, as described previously (Fukunaga et al., 2010).

\section{Iron staining}

Iron staining was done using a modified Perls iron staining as described previously (Carlson et al., 2009). Briefly, free floating sections of a P30 control brain and the P30 iron extracted brain were simultaneously incubated for $5 \mathrm{~min}$ in 10\% potassium ferrocyanide (Sigma 
Aldrich). Then, they were incubated in a freshly made Perls solution for $20 \mathrm{~min}$. The Perls reaction was intensified with 3,3' diaminobenzidine (DAB) during $10 \mathrm{~min}$. Sections were then rinsed in PBS and submerged in increasing concentrations of ethyl alcohol before being immersed in a clearing agent, air-dried and coverslipped. Control sections were incubated in Perls solution with phosphate buffered saline (PBS) substituted for potassium ferrocyanide, and were negative for any DAB staining.

\section{Post-processing}

The phase images $(\phi)$ were first unwrapped (Goldstein et al., 1988 ), and subsequently converted frequency shifts in ppm, $\Delta \omega$, using the following relationship:

$\Delta \omega=\phi /(\mathrm{TE}) /\left(\gamma \mathrm{B}_{0}\right) 10^{6}$

where $\gamma$ is the proton gyromagnetic constant and $B_{0}$ is the main magnetic field. Such a notation has the advantage to provide results that are field independent, thus allowing the integration of results from different magnet field strengths and performed at different echo times.

Large scale phase shifts due to magnetic susceptibility differences between air and tissue and imperfect shimming were removed using the SHARP algorithm (Schweser et al., 2011). In our implementation of the SHARP method, because of the 2D nature of our acquisition with thick slices $(0.5 \mathrm{~mm})$ when compared to the in-plane resolution (0.6-0.1 mm), the phase data was first interpolated in the $\mathrm{z}$ - direction to quasi-isotropic resolution. The SHARP method was then applied using a spherical kernel with a $\cos ^{2}$ modulation and a radius 4 times the lower spatial resolution. The spherical kernel was defined in a higher resolution space (4 times the resolution of the phase data) and subsequently brought to the lower resolution while taking into account the partial volume effects. The deconvolution mask was defined by convolving the original mask with the spherical kernel and taking all the values with an intensity greater than 0.99. The deconvolution was performed with a singular value decomposition truncation, as in the original implementation (Schweser et al., 2011), at 0.4

For illustration purposes the encoding of contrast on phase images was chosen to match the staining identified by Black Gold with white matter (negative frequency shifts) appearing dark. The focus of this study was on two prominent white matter structures: the corpus callosum (CC, at the level of the head of the hippocampus); the limbs of the anterior commissure (AC). Frequency shifts differences were measured between hand drawn regions of interest in those white matter structures and surrounding gray matter.

Quantitative maps of the apparent diffusion coefficient, the axial diffusivity and the radial diffusivity were derived using software written in Matlab (MathWorks, Natick, Ma, USA) as described previously (Song et al., 2002, 2003, 2005; Sun et al., 2003). Regions of interest were placed using Image (Abramoff et al., 2004) on the corpus callosum from the RGB map as described by Lodygensky et al. (2010).

\section{Statistics}

All data are presented as mean \pm standard error of the mean (SEM). Comparisons between groups were performed using a Mann-Whitney test. Statistical significance was set at 0.05. Correlation analysis was done using the Spearman non-parametric correlation method. Analyses were performed using GraphPad Prism (GraphPad Software, La Jolla, CA).

\section{Results}

\section{Myelination on the developing brain}

To determine the effect of developmental myelination on phase contrast, phase imaging was acquired at P3, P4, P15, P21 and P40 and compared to myelination assessed by histology. Phase images and histology slices showing the corpus callosum and the limbs of the anterior commissure of representative animals at different stages

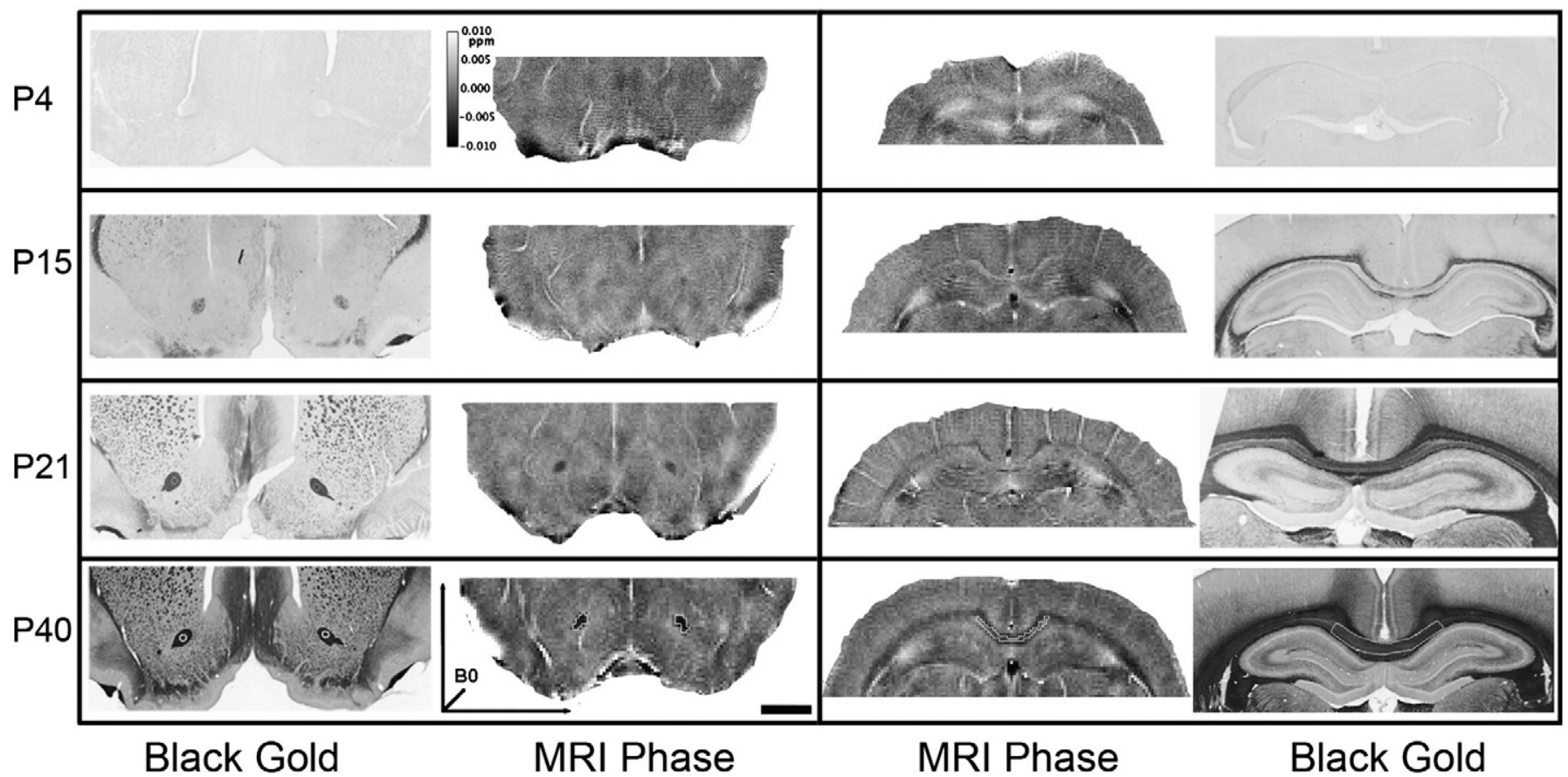

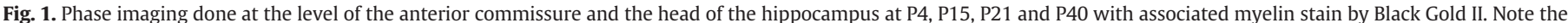

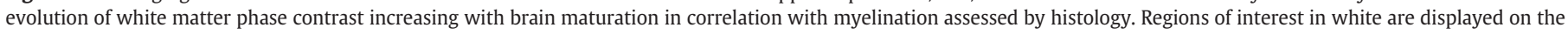
P40 brain. Bar $2 \mathrm{~mm}$ 
A
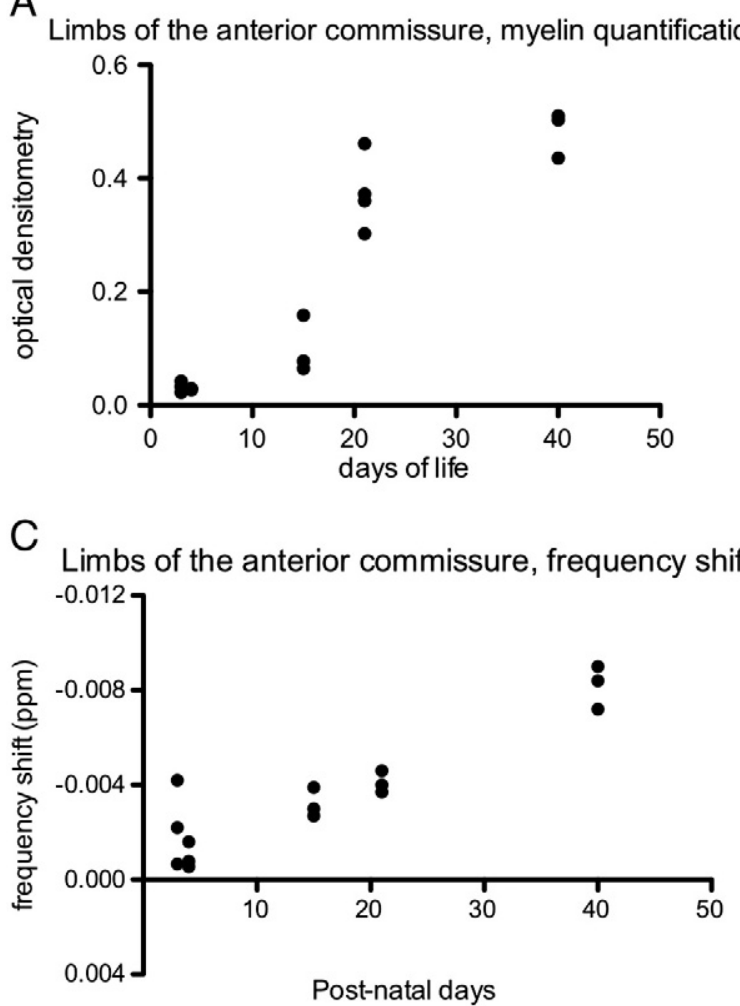

$E$

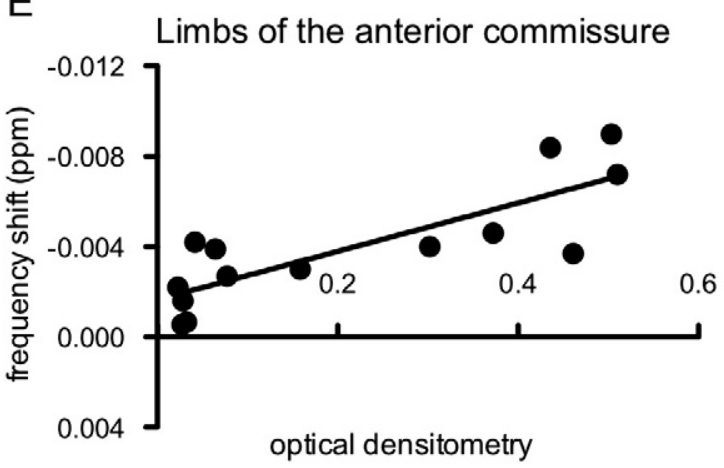

$B$

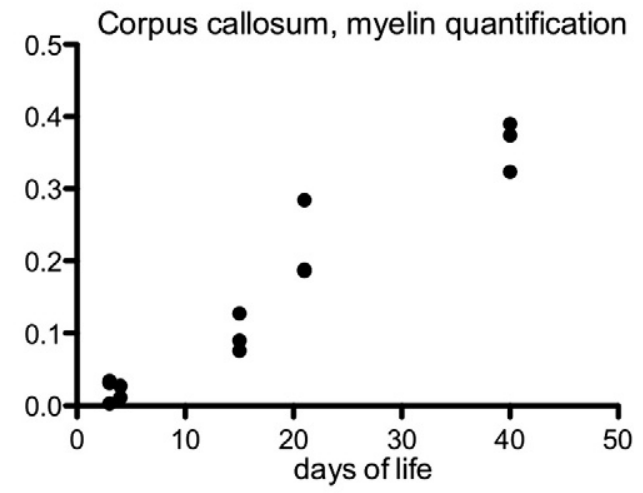

D Corpus callosum, frequency shift

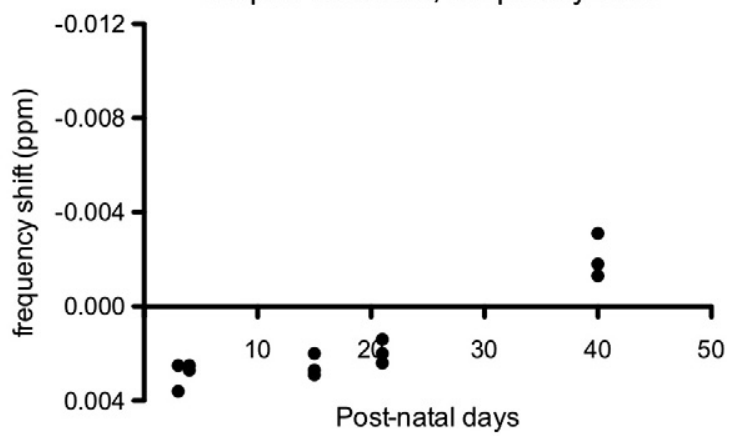

$\mathrm{F}$

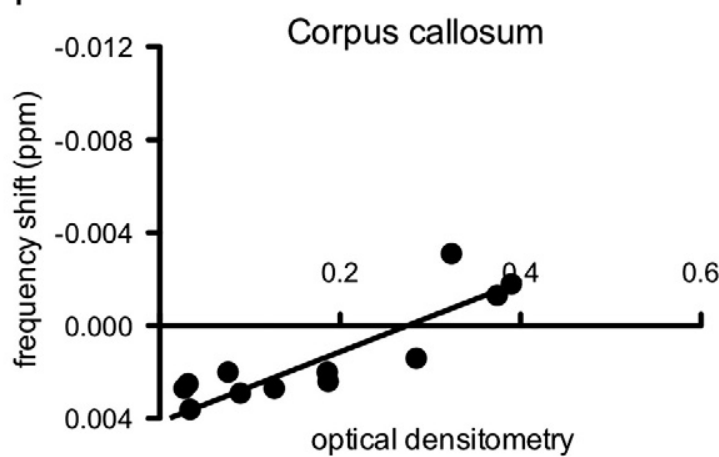

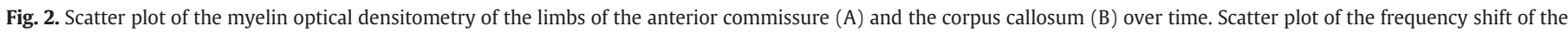

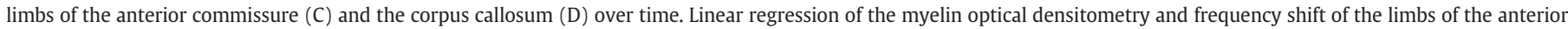

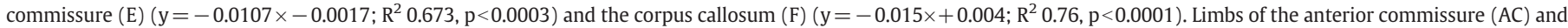
corpus callosum (CC).

of development are shown in Fig. 1. Vessels perpendicular to the $\mathrm{B}_{0}$ field were seen both on rat and mice in vivo images similar to images obtained on adult rats at $14.1 \mathrm{~T}$ (Marques et al., 2009). A quantitative assessment of myelination by Black Gold in the anterior commissure and the corpus callosum was performed (Fig. 2), showing that, at P3 and $\mathrm{P} 4$, the brains are essentially non-myelinated. The initiation of myelination starts at approximately P15, increasing at P21, with abundant myelin by $\mathrm{P} 40$. The contrast observed in phase imaging (Fig. 1) followed the same progression, distinguishing myelinated white matter structures from non-myelinated ones. A clear anatomical and temporal agreement of the myelinated brain structures was observed between the in vivo phase imaging and ex vivo myelin staining. Note that at developmental stages where myelin was essentially absent, both the CC and the AC were initially not distinguishable from surrounding regions and became more apparent during brain maturation. The two measurements were significantly correlated (Figs. 2E-F) both in the corpus callosum and the limbs of the anterior commissure as implied by the $\mathrm{R}^{2}$ of the linear regression fit of 0.67 and 0.77 for the AC and CC respectively. The linear correlations for each of these regions had similar values $(-0.011 \pm 0.002$ and $-0.015 \pm 0.002$ for the $\mathrm{AC}$ and $\mathrm{CC}$ ), while significantly different phase offsets were found for the case where no myelin/black gold was present ( $\mathrm{y}$-crossing points for $\mathrm{x}=0$ were $-0.0017 \pm 0.0006$ and $0.0042 \pm 0.0005 \mathrm{ppm}$ for the AC and CC respectively).

\section{Myelin quantification in shiverer mice}

To further determine whether phase imaging can reflect myelin content, we compared a model of dysmyelination (shiverer mice) to age-matched control mice using aforementioned histology in vivo MRI as well as DTI.

Optimal shimming particularly at high magnetic fields was found to be particularly difficult in mice due to their small dimensions and 

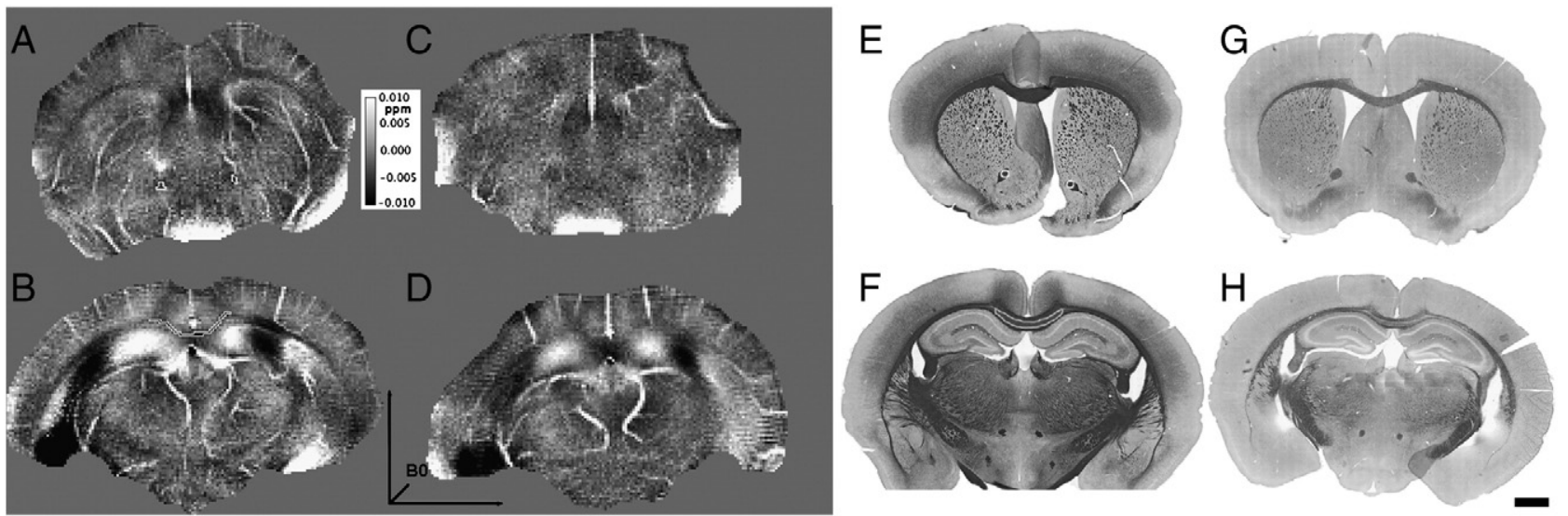

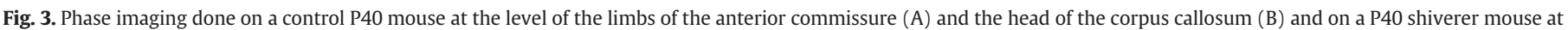

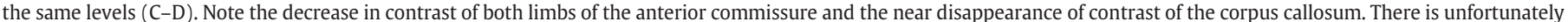

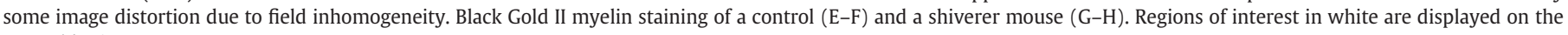
control brain. Bar $1 \mathrm{~mm}$.

explains the increased signal dropout in parts of the cerebral cortex in mice (Fig. 3) when compared to rat images (Fig. 1).

Phase images of shiverer mice exhibited drastically reduced WMGM contrast compared to control mice (Fig. 3). When the frequency shift was quantified, a decrease of $66 \%$ for the corpus callosum $(p<0.05)$ and a 59\% decrease of the limbs of the anterior commissure $(p<0.05)$ were observed in the shiverer mice when compared to control animals (Fig. 4).

The histological evaluation of myelination with Black Gold II showed a strikingly similar statistically significant difference in optical densitometry of $60 \%$ in the anterior commissure of shiverer mice, as well as a $61 \%$ decrease in the corpus callosum compared to control mice.

An $18 \%$ increase in radial diffusivity was found in the corpus callosum of shiverer mice that did not reach statistical significance $(p<0.095)$ compared to control mice (Fig. 5).

\section{Effect of iron extraction on phase contrast}

To distinguish the effect of myelin on phase contrast from iron content, phase images of ex vivo brain samples were acquired before and after extraction of tissue iron on two P30 rat brain, tested by modified Perls staining. In Fig. 6 it is possible to see that the iron extraction was achieved, as evaluated by Pearl staining, while myelin contrast, as assessed by Black Gold remained unchanged. The phase images though, have their gray/white matter contrast unaffected by the iron extraction protocol showing that iron does not play a major role towards the observed contrast (Fig. 6). Note that when the ex vivo experiment on the first rat brain (Fig. 6A) was performed with the anterior posterior direction positioned perpendicular to the main magnetic field, the observed contrast was dramatically different from that observed in vivo (Figs. 1 and 3). A clear dipole shape was visible surrounding the $\mathrm{AC}$, which is expected to be seen when a
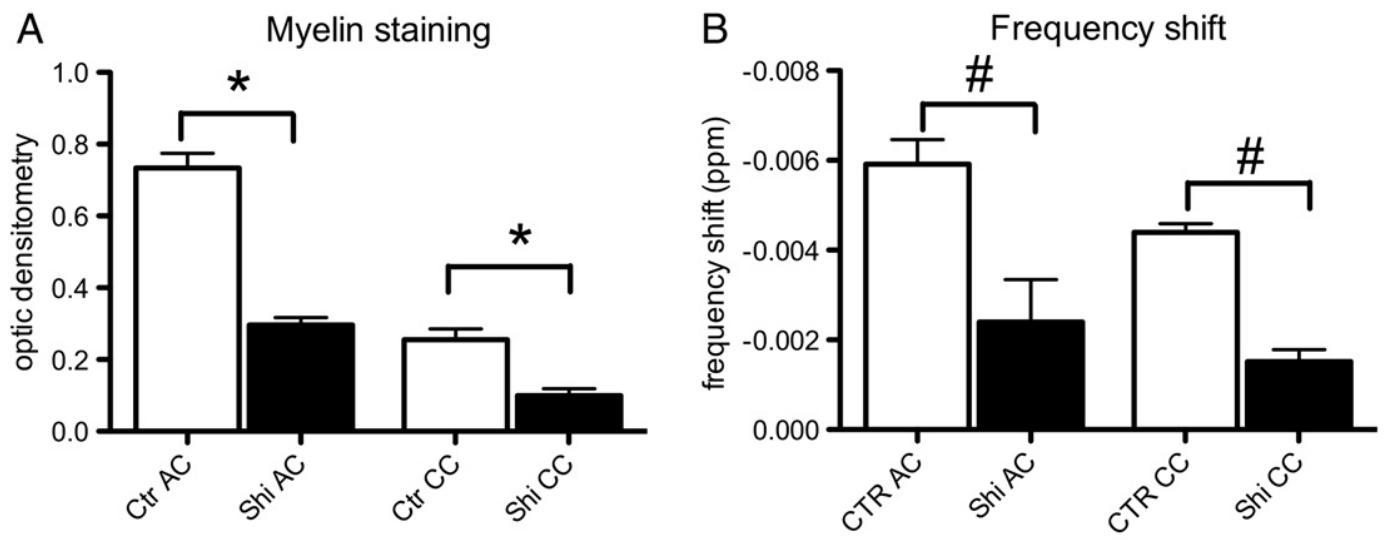

\begin{tabular}{l|ll}
\hline & $\begin{array}{l}\text { Myelin staining } \\
\text { Optic densitometry }\end{array}$ & $\begin{array}{l}\text { Frequency shift } \\
(\text { ppm })\end{array}$ \\
\hline Ctr AC & $0.733 \pm 0.04$ & $-0.0059 \pm 0.0005$ \\
Shiv AC & $0.297 \pm 0.02$ & $-0.0024 \pm 0.00095$ \\
\hline Ctr CC & $0.26 \pm 0.03$ & $-0.0044 \pm 0.00019$ \\
Shi CC & $0.099 \pm 0.02$ & $-0.0015 \pm 0.00026$ \\
\hline
\end{tabular}

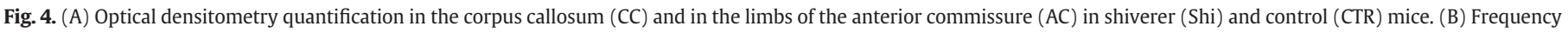
shift quantification in the corpus callosum and in the limbs of the anterior commissure of shiverer and control mice. ${ }^{*} \mathrm{p}<0.01 ; \# \mathrm{p}<0.05$. 


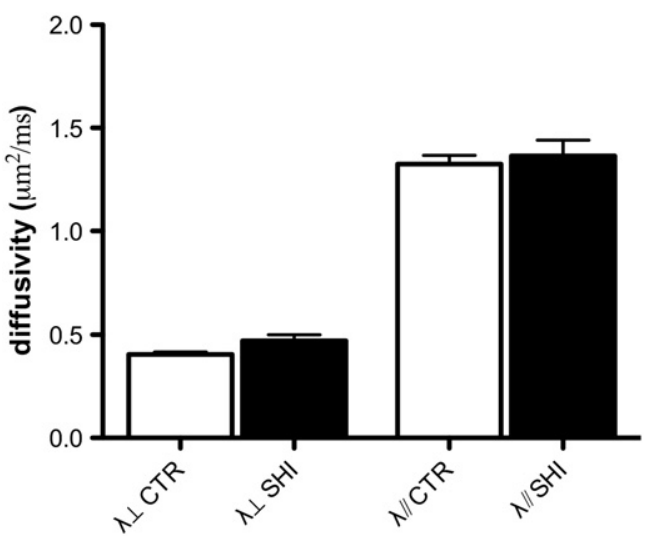

Fig. 5. In vivo diffusivity measurements of the corpus callosum in control and shiverer mice. Control radial diffusivity $\left(\lambda \perp\right.$ CTR) $0.41 \pm 0.01 \mu \mathrm{m}^{2} / \mathrm{ms}$, shiverer radial diffusivity $(\lambda \perp$ SHI $) 0.47 \pm 0.03 \mu \mathrm{m}^{2} / \mathrm{ms}, \mathrm{p}=0.095$. Control axial diffusivity $(\lambda / / \mathrm{CTR}) 1.32 \pm$ $0.04 \mu \mathrm{m}^{2} / \mathrm{ms}$, shiverer axial diffusivity $(\lambda / / \mathrm{SHI}) 1.36 \pm 0.08 \mu \mathrm{m}^{2} / \mathrm{ms}, \mathrm{p}=1$.

long structure with a magnetic susceptibility is positioned perpendicular to the applied magnetic field (Marques and Bowtell, 2005). Also, the contrast of the external commissure changes polarity with respect to the remaining gray matter when its angle to the main magnetic field goes from parallel to perpendicular. Fig. 6B shows a second data set where the experiments were performed with the anterior posterior axis along the main magnetic field. In this case the contrast observed was comparable with the phase images obtained in vivo.

\section{Discussion}

We characterized the ability of phase imaging to detect and quantify myelin in vivo at high resolution. The evolution of gray/white matter phase contrast during development was in good agreement with known evolution of myelination (Hamano et al., 1996), with P3 and P4 rat pups having essentially un-myelinated brains, initiation of myelination at P15, readily identifiable at P21 and abundant at P40. The myelination process was evaluated by histology (Figs. 1 and 2) and a significant correlation was found between the two.

Phase imaging is known to depend not only on the shape of the different structures with different susceptibilities (Marques and Bowtell, 2005; Salomir et al., 2003; Schafer et al., 2009) but also on local microstructure (He and Yablonskiy, 2009), on local anisotropy of the magnetic susceptibility (Lee et al., 2010b; Liu, 2010) and even on nonsusceptibility mechanisms such as water exchange mechanisms (Luo et al., 2010; Shmueli et al., 2011; Zhong et al., 2011). In this study the phase shift observed locally between adjacent structures that remain geometrically unchanged during development was used to estimate myelination. Two structures, spatially very distinct, and with WM tracts oriented with $\sim 90^{\circ}$ from one another (AC parallel to B0, CC perpendicular to $\mathrm{B}_{0}$ ) were studied and it was observed that the frequency shift in each structure had a high degree of correlation with myelin and could therefore in the future allow myelin quantification in those structures.

To further test for the sensitivity of phase contrast to myelin we investigated this technique in a model of dysmyelination seen in shiverer mice in vivo. DTI studies demonstrated that the CC and AC main direction of diffusivity remains unchanged, with a comparable increase of in vivo radial diffusivity of the shiverer corpus callosum (although statistical significance was not reached) to the results described ex vivo (Ou et al., 2009). When analyzing the relevance of phase imaging to assess dysmyelination on the same animals we found significant change in frequency shift corresponding with changes seen in histology (Figs. 3 and 4). Interestingly similar sensitivity of phase contrast to myelin injury was shown in mice exposed to cuprizone (Baxan et al., 2010) and in ex vivo shiverer mice (Liu et al., 2011) thus supporting the relevance of phase contrast imaging to assess myelin integrity.

Although the in vivo data shown indicates that myelin has a dominant role towards the observed contrast between gray and white matter, it should be noticed that iron deposition is known to modulate myelin contrast (Fukunaga et al., 2010). Iron has been shown in humans to be correlated to the phase contrast between deep gray matter structures such as the subtantia nigra, red nucleus, globus pallidus and putamen (Schweser et al., 2011) (known to be extremely rich in iron concentration) and the surrounding white matter. It has also been shown to be responsible for some intracortical gray matter phase contrast (Fukunaga et al., 2010). Inspired by the work of Fukunaga et al. that performed iron extraction by reductive dissolution to demonstrate the role of iron towards intracortical gray matter contrast, we performed a similar study to assess ex vivo the potential iron contribution to the observed contrast. Two ex vivo P30 rat brain were imaged before and after iron extraction and were processed for histology both for myelin and iron quantification. The histological data was compared to that performed on two control P30 rat brain to verify that myelin wasn't severely affected while iron was clearly reduced by the iron extraction methodology. Fig. 6 demonstrates that the phase contrast of myelin was unaffected by iron extraction in both animals. The observation of the very distinctive patterns in the ex vivo brain scan that was scanned with the anterior posterior axis perpendicular to the main magnetic field is a confirmation of the susceptibility origin of the white gray matter contrast observed in white matter as well as of the inherent limitations of assessing myelination from raw phase data.

Overall, the present study shows that both during development and in the dysmyelinating shiverer mice models, the gray white matter contrast observed in phase images is correlated to their myelin content. One conclusion that can be therefore drawn is that only structures associated with myelin are likely to impact the phase contrast observed between white and gray matter. Other contributions such as the axonal microstructure (He and Yablonskiy, 2009) per se are expected to have a smaller (but not negligible) impact in the contrast. The upper limit of these other contributions towards the final contrast could be estimated from the $\mathrm{y}$ axis crossing point in Figs. 2E and F. In particular case of the AC the zero crossing (located at $-0.0017 \mathrm{ppm}$ ) when compared to the frequency shift variation from the fully un-myelinated to the fully myelinated brain (the myelin correlated contribution is approximately $-0.006 \mathrm{ppm}$ ) suggests that $\sim 20 \%$ of the contrast between gray and white matter is not myelin related. The analysis of the CC is more complex due to the presence of neighboring white matter structures that have different maturation timelines and could affect the phase shift observed between the CC and gray matter. One possible mechanism that could explain the different polarities of the offsets observed in the ac and the $\mathrm{cc}$ in the absence of myelin would be an axonal microstructure contribution to the contrast (He and Yablonskiy, 2009). Against this hypothesis is the observation that the offset is greater in magnitude in the corpus callosum (which is perpendicular to the main magnetic field) than in the anterior commissure (which is close to parallel to the magnetic field $\sim 10-20^{\circ}$ ). Such a modulation is inconsistent with a recently proposed local microstructure contribution ( $\mathrm{He}$ and Yablonskiy, 2009) that would be characterized by a term proportional to $\cos ^{2}(\theta)-1 / 3$ if it was assumed that the underlying microstructure of the AC and the CC fiber bundles would be exactly the same. The second mechanism that could explain the variation of polarity of the white gray matter contrast in the absence of myelin would be a variation of the local bulk susceptibility of the surrounding gray matter or of the white matter itself. A change of the bulk susceptibility between the CC and the AC could also be explained by susceptibility anisotropy. In this case, a change in polarity of the sign of $\left(\chi_{\mathrm{wm}}^{\|}-\chi_{\mathrm{GM}}\right)$ in respect to $\left(\chi_{\mathrm{wm}}^{\frac{1}{\mathrm{~m}}}-\chi_{\mathrm{GM}}\right)$ would be necessary, where $\chi_{\mathrm{wm}}^{\| 1}$ and $\chi_{\mathrm{wm}}^{\frac{1}{\mathrm{~m}}}$ represent the component of susceptibility parallel and perpendicular 

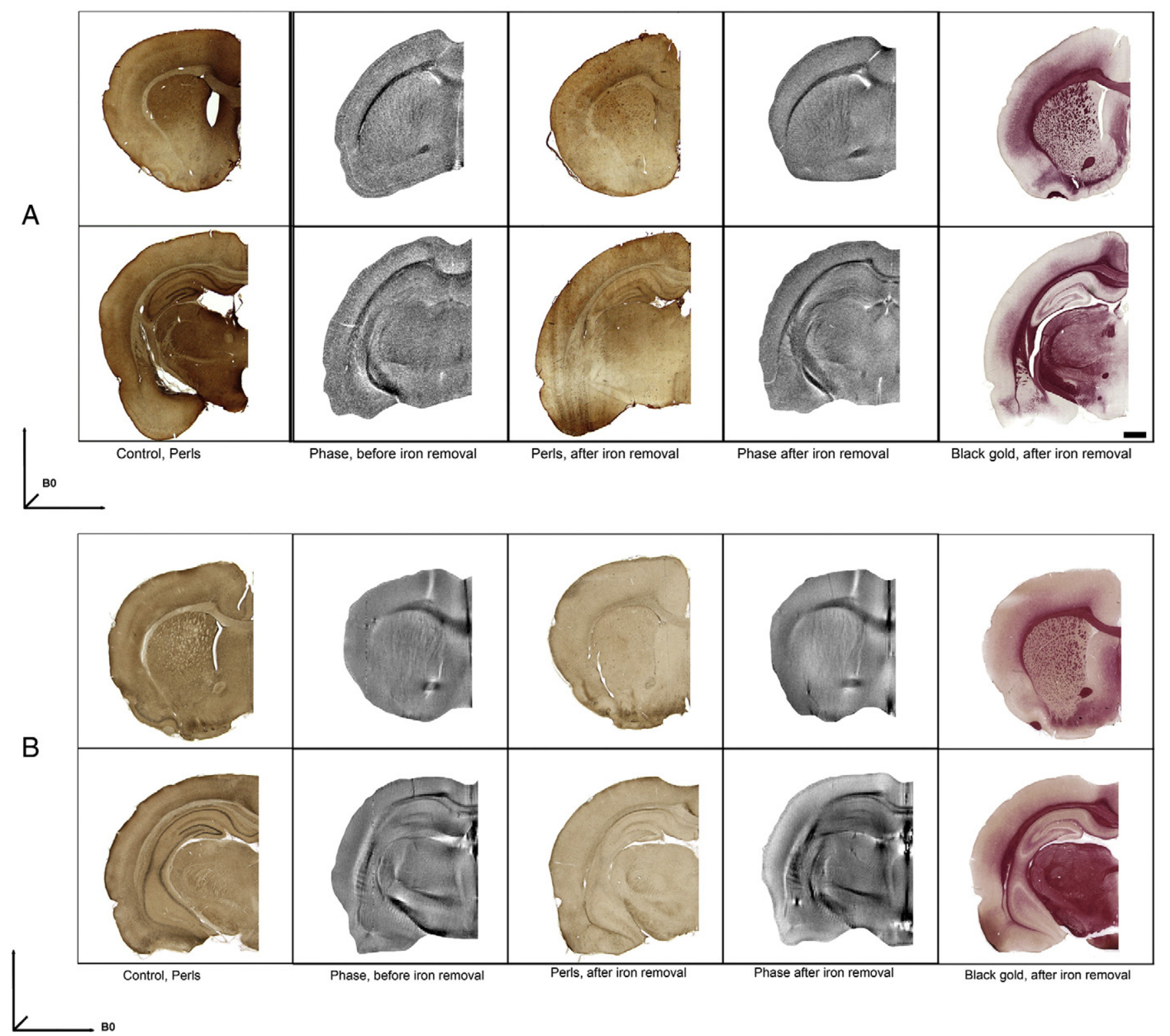

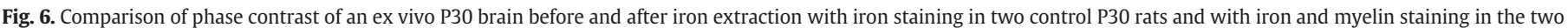

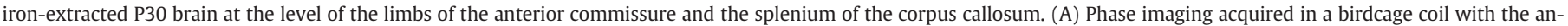

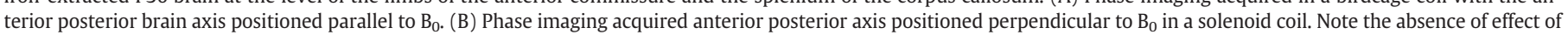

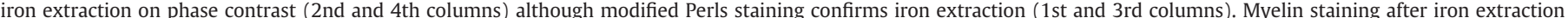

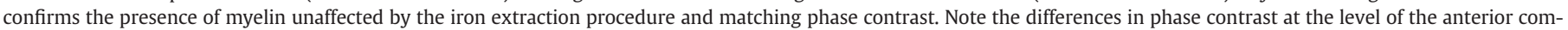
missure between the acquisition where this structure is parallel to $\mathrm{B}_{0}$ and the one done perpendicular to $\mathrm{B}_{0}$ with a "dipole" shape surrounding the AC in the later. Bar 1 mm.

to the main magnetic field and $\chi_{\mathrm{GM}}$ represents the susceptibility of gray matter (that was found to be isotropic). This was not the case in the adult brain (Liu, 2010) but it could be in the immature brain assuming that the observed susceptibility tensor would be the addition of two susceptibility tensors, one associated with myelin (which would not be observable in the unmyelinated brain) and one associated with other organized structures (where the sign of $\left(\chi_{\mathrm{wm}}^{\|}-\chi_{\mathrm{GM}}\right)$ is different $\left.\left(\chi_{\mathrm{wm}}^{\frac{1}{m}}-\chi_{\mathrm{GM}}\right)\right)$. So far the chemical shift contribution towards phase imaging has only been proposed as an isotropic effect (Luo et al., 2010; Shmueli et al., 2011; Zhong et al., 2008) and therefore could not be responsible for the observed polarity change between the ac and the cc in the absence of myelin. It could be hypothesized that chemical shift could have a contribution that is structure orientation dependent (macromolecules can be arranged in an ordered fashion resulting phases depending on the orientation of the underlying structures) and such an anisotropic mechanism could not be differentiated (in our data) from the effect described by He et al. (He and Yablonskiy, 2009). The study that most directly observed the exchange contribution towards phase contrast in brain tissues (in ex vivo tissue samples) showed no significant anisotropies despite the different white matter orientations present in the tissue samples (Shmueli et al., 2011). Furthermore, at $X=0$ there is no myelin, which significantly reduces the pool responsible for chemical shifts associated to amide and $(\mathrm{NH})$ and hydroxyl $(\mathrm{OH})$ groups (Shmueli et al., 2011) and hence chemical shift is unlikely to be responsible for this effect.

The only study that has in a more systematic fashion addressed the role of microstructure orientation has been carried in humans (Denk et al., 2011) observing the phase dependence as a function of the fibers orientation. In our work we observed that the fiber orientation dependence has a cause that is not related to myelin distribution. Our observation in premyelinated brain could therefore be one of the first in 
vivo demonstration of the role of local microstructure, and would suggest that this effect is not dominated by structures related to the myelin packing but rather to other structures with an orientation that correlates with that of the axons (for example, proteins associated to the axonal structure - such as neurofilaments and microtubules - or the axonal wall). This hypothesis assumes that there are no significant changes with development and region for gray matter, used as reference. The observation of different polarity of the zero crossing could also be hypothetically explained by having very different iron or calcium concentration differences between the surrounding gray matter and white matter structures at early stages of development.

The back calculation of susceptibility maps (de Rochefort et al., 2010; Liu et al., 2009) from the measured frequency shift maps would potentially allow the quantification of myelination. Although such techniques have been used with some success to quantify contrast agents, or the iron content of deep gray matter structures, its application should be made with care in the context of gray-white matter contrast. For example, as observed in this paper, contrast that is microstructure orientation dependent either via the Lorentz "cylinder" (He and Yablonskiy, 2009) or the anisotropy of the susceptibility tensor (Liu, 2010) exists and should be introduced in the model. The local frequency shifts should only be used as mean to quantify susceptibility and myelination when the full mechanism that translates myelin content into phase contrast is known or in the case when a group study is first done to calibrate the local relationship between frequency shift and myelination for different brain regions as done in this study. A quantitative measure of myelination would be of great importance in pre-clinical and clinical applications such as monitoring response to treatment in periventricular leukomalacia and in multiple sclerosis.

\section{Conclusions}

As the geometry of cerebral white matter and most of the underlying structure is conserved during development or disease, it is possible to state, based on the present data that the measurement of local frequency shift allows the assessment of local myelination both in development and disease. Furthermore it is possible to conclude that contributions to the phase contrast between white and gray matters are not confined to myelin and that other organized structures play a role even if not dominant.

\section{Acknowledgments}

This study was supported by Swiss National Fund 33CM30124101 and 132821, by CIBM of the UNIL, UNIGE, HUG, CHUV, EPFL and the Boninchi, De Reuter, Leenaards and Jeantet Foundations. The authors are grateful to Yves Pilloud for his contribution in building the coil used on mice and the MRI compatible rodent head holder.

\section{References}

Abramoff, M.D., Magelhaes, P.J., Ram, S.J., 2004. Image processing with image. J. Biophotonics Int. 11, 36-42.

Baxan, N., Harsan, L.A., Dragonu, I., Hennig, A., von Elverfeldt, D., 2010. Myelin as a primary source of phase contrast demonstrated in vivo in the mouse brain. Proc., ISMRM, 19th Annual Meeting and Exhibition, Stockholm, Sweden, p. 3016.

Beaulieu, C., Fenrich, F.R., Allen, P.S., 1998. Multicomponent water proton transverse relaxation and T2-discriminated water diffusion in myelinated and nonmyelinated nerve. Magn. Reson. Imaging 16, 1201-1210.

Carlson, E.S., Tkac, I., Magid, R., O'Connor, M.B., Andrews, N.C., Schallert, T., Gunshin, H., Georgieff, M.K., Petryk, A., 2009. Iron is essential for neuron development and memory function in mouse hippocampus. J. Nutr. 139, 672-679.

Cheng, Y.C., Neelavalli, J., Haacke, E.M., 2009. Limitations of calculating field distributions and magnetic susceptibilities in MRI using a Fourier based method. Phys. Med. Biol. 54, 1169-1189.

Chernoff, G.F., 1981. Shiverer: an autosomal recessive mutant mouse with myelin deficiency. J. Hered. 72, 128

Cowan, F.M., de Vries, L.S., 2005. The internal capsule in neonatal imaging. Semin. Fetal Neonatal Med. 10, 461-474. de Rochefort, L., Brown, R., Prince, M.R., Wang, Y., 2008. Quantitative MR susceptibility mapping using piece-wise constant regularized inversion of the magnetic field. Magn. Reson. Med. 60, 1003-1009.

de Rochefort, L., Liu, T., Kressler, B., Liu, J., Spincemaille, P., Lebon, V., Wu, J., Wang, Y. 2010. Quantitative susceptibility map reconstruction from MR phase data using bayesian regularization: validation and application to brain imaging. Magn. Reson. Med. 63, 194-206.

Denk, C., Torres, E.H., Mackay, A., Rauscher, A., 2011. The influence of white matter fibre orientation on MR signal phase and decay. NMR Biomed. 24 (3), 246-252.

Deoni, S.C., Rutt, B.K., Arun, T., Pierpaoli, C., Jones, D.K., 2008. Gleaning multicomponent T1 and T2 information from steady-state imaging data. Magn. Reson. Med. 60, $1372-1387$.

Deoni, S.C., Mercure, E., Blasi, A., Gasston, D., Thomson, A., Johnson, M., Williams, S.C., Murphy, D.G., 2011. Mapping infant brain myelination with magnetic resonance imaging. J. Neurosci. 31, 784-791.

Du, Y.P., Chu, R., Hwang, D., Brown, M.S., Kleinschmidt-DeMasters, B.K., Singel, D. Simon, J.H., 2007. Fast multislice mapping of the myelin water fraction using multicompartment analysis of T 2* decay at 3T: a preliminary postmortem study. Magn. Reson. Med. 58, 865-870.

Duyn, J.H., van Gelderen, P., Li, T.Q., de Zwart, J.A., Koretsky, A.P., Fukunaga, M., 2007. High-field MRI of brain cortical substructure based on signal phase. Proc. Natl. Acad. Sci. U.S.A. 104, 11796-11801.

Fukunaga, M., Li, T.Q., van Gelderen, P., de Zwart, J.A., Shmueli, K., Yao, B., Lee, J., Maric, D., Aronova, M.A., Zhang, G., Leapman, R.D., Schenck, J.F., Merkle, H., Duyn, J.H., 2010. Layer-specific variation of iron content in cerebral cortex as a source of MRI contrast. Proc. Natl. Acad. Sci. U.S.A. 107, 3834-3839.

Goldstein, R.M., Zebker, H.A., Werner, C.L., 1988. Satellite radar interferometry - twodimensional phase unwrapping. Radio Sci. 23, 713-720.

Gruetter, R., 1993. Automatic, localized in vivo adjustment of all first- and secondorder shim coils. Magn. Reson. Med. 29, 804-811.

Hamano, K., Iwasaki, N., Takeya, T., Takita, H., 1996. A quantitative analysis of rat central nervous system myelination using the immunohistochemical method for MBP. Brain Res. Dev. Brain Res. 93, 18-22.

He, X., Yablonskiy, D.A., 2009. Biophysical mechanisms of phase contrast in gradient echo MRI. Proc. Natl. Acad. Sci. U.S.A. 106, 13558-13563.

Hwang, D., Kim, D.H., Du, Y.P., 2010. In vivo multi-slice mapping of myelin water content using T2* decay. Neuroimage 52, 198-204.

Jara, H., Wehrli, F.W., 1994. Determination of background gradients with diffusion MR imaging. J. Magn. Reson. Imaging 4, 787-797.

Lancaster, J.L., Andrews, T., Hardies, L.J., Dodd, S., Fox, P.T., 2003. Three-pool model of white matter. J. Magn. Reson. Imaging 17, 1-10.

Lee, J., Hirano, Y., Fukunaga, M., Silva, A.C., Duyn, J.H., 2010a. On the contribution of deoxyhemoglobin to MRI gray-white matter phase contrast at high field. Neuroimage 49, 193-198.

Lee, J., Shmueli, K., Fukunaga, M., van Gelderen, P., Merkle, H., Silva, A.C., Duyn, J.H. 2010b. Sensitivity of MRI resonance frequency to the orientation of brain tissue microstructure. Proc. Natl. Acad. Sci. U.S.A. 107, 5130-5135.

Liu, C., 2010. Susceptibility tensor imaging. Magn. Reson. Med. 63, 1471-1477.

Liu, T., Spincemaille, P., de Rochefort, L., Kressler, B., Wang, Y., 2009. Calculation of susceptibility through multiple orientation sampling (COSMOS): a method for conditioning the inverse problem from measured magnetic field map to susceptibility source image in MRI. Magn. Reson. Med. 61, 196-204.

Liu, C., Li, W., Johnson, G.A., Wu, B., 2011. High-field (9.4 T) MRI of brain dysmyelination by quantitative mapping of magnetic susceptibility. Neuroimage 56 (3), 930-938.

Lodygensky, G.A., West, T., Stump, M., Holtzman, D.M., Inder, T.E., Neil, J.J., 2010. In vivo MRI analysis of an inflammatory injury in the developing brain. Brain Behav. Immun. 24, 759-767.

Luo, J., He, X., d'Avignon, D.A., Ackerman, J.J., Yablonskiy, D.A., 2010. Protein-induced water $1 \mathrm{H}$ MR frequency shifts: contributions from magnetic susceptibility and exchange effects. J. Magn. Reson. 202, 102-108.

MacKay, A., Whittall, K., Adler, J., Li, D., Paty, D., Graeb, D., 1994. In vivo visualization of myelin water in brain by magnetic resonance. Magn. Reson. Med. 31, 673-677.

Marques, J.P., Bowtell, R., 2005. Application of a fourier-based method for rapid calculation of field inhomogeneity due to spatial variation of magnetic susceptibility. Concepts Magn. Reson. B Magn. Reson. Eng. 25B, 65-78.

Marques, J.P., Maddage, R., Mlynarik, V., Gruetter, R., 2009. On the origin of the MR image phase contrast: an in vivo MR microscopy study of the rat brain at $14.1 \mathrm{~T}$. Neuroimage 46, 345-352.

Ou, X., Sun, S.W., Liang, H.F., Song, S.K., Gochberg, D.F., 2009. The MT pool size ratio and the DTI radial diffusivity may reflect the myelination in shiverer and control mice. NMR Biomed. 22, 480-487.

Privat, A., Jacque, C., Bourre, J.M., Dupouey, P., Baumann, N., 1979. Absence of the major dense line in myelin of the mutant mouse "shiverer". Neurosci. Lett. 12, 107-112.

Salomir, R., de Senneville, B.D., Moonen, C.T.W., 2003. A fast calculation method for magnetic field inhomogeneity due to an arbitrary distribution of bulk susceptibility. Concepts Magn. Reson. B Magn. Reson. Eng. 19B, 26-34.

Schafer, A., Wharton, S. Gowland, P., Bowtell, R, 2009. Using magnetic field simulation to study susceptibility-related phase contrast in gradient echo MRI. Neuroimage 48 126-137.

Schmued, L., Bowyer, J., Cozart, M., Heard, D., Binienda, Z., Paule, M., 2008. Introducing Black-Gold II, a highly soluble gold phosphate complex with several unique advantages for the histochemical localization of myelin. Brain Res. 1229, 210-217.

Schweser, F., Deistung, A., Lehr, B.W., Reichenbach, J.R., 2011. Quantitative imaging of intrinsic magnetic tissue properties using MRI signal phase: an approach to in vivo brain iron metabolism? Neuroimage 54, 2789-2807. 
Shmueli, K., de Zwart, J.A., van Gelderen, P., Li, T.Q., Dodd, S.J., Duyn, J.H., 2009. Magnetic susceptibility mapping of brain tissue in vivo using MRI phase data. Magn. Reson. Med. 62, 1510-1522.

Shmueli, K., Dodd, S.J., Li, T.Q., Duyn, J.H., 2011. The contribution of chemical exchange to MRI frequency shifts in brain tissue. Magn. Reson. Med. 65, 35-43.

Song, S.K., Sun, S.W., Ramsbottom, M.J., Chang, C., Russell, J., Cross, A.H., 2002. Dysmyelination revealed through MRI as increased radial (but unchanged axial) diffusion of water. Neuroimage 17, 1429-1436.

Song, S.K., Sun, S.W., Ju, W.K., Lin, S.J., Cross, A.H., Neufeld, A.H., 2003. Diffusion tensor imaging detects and differentiates axon and myelin degeneration in mouse optic nerve after retinal ischemia. Neuroimage 20, 1714-1722.
Song, S.K., Yoshino, J., Le, T.Q., Lin, S.J., Sun, S.W., Cross, A.H., Armstrong, R.C., 2005. Demyelination increases radial diffusivity in corpus callosum of mouse brain. Neuroimage 26, 132-140.

Sun, S.W., Neil, J.J., Song, S.K., 2003. Relative indices of water diffusion anisotropy are equivalent in live and formalin-fixed mouse brains. Magn. Reson. Med. 50, 743-748.

Whittall, K.P., MacKay, A.L., 1989. Quantitative interpretation of NMR relaxation data. J. Magn. Reson. 84, 134-152.

Zhong, K., Leupold, J., von Elverfeldt, D., Speck, O., 2008. The molecular basis for gray and white matter contrast in phase imaging. Neuroimage 40, 1561-1566.

Zhong, K., Ernst, T., Buchthal, S., Speck, O., Anderson, L., Chang, L., 2011. Phase contrast imaging in neonates. Neuroimage 55 (3), 1068-1072. 